\title{
La Revolución cubana de 1895. El aporte de los viajes de Arístides Agüero a Perú, Chile y Bolivia.
}

\author{
The Cuban Revolution of 1895. The contribution of Arístides \\ Agüero's trips to Peru, Chile and Bolivia.
}

\author{
Claudio Antonio Gallegos \\ Instituto de Investigaciones Económicas y Sociales del Sur; \\ Concejo Nacional de Investigaciones Científicas y Técnicas; \\ Departamento de Economía, \\ Universidad Nacional del Sur (Argentina) \\ cgallegos80@gmail.com
}

\begin{abstract}
Resumen
El presente artículo tiene como objetivo analizar las redes desarrolladas por el Partido Revolucionario Cubano sobre Perú, Chile y Bolivia, con la intención de lograr apoyo a la insurrección cubana de 1895. Dentro de los estudios sobre la guerra de 1895 se evidencia una ausencia en lo que respecta a relaciones diplomáticas por parte de la República de Cuba. A través de correspondencia pública y privada se demuestra, entonces, como uno de los delegados de dicho partido, Arístides Agüero, construye relaciones de poder por medio de diversas acciones sobre la Región Andina Sur. Considerada la guerra como un hecho social complejo recurrente en la historia de la humanidad -desde una mirada sociológica y geopolítica-, se busca describir el impacto de dichas redes localizando sus ámbitos de realización, ya sean organizacionales, políticos o bélicos. Asimismo, se busca complementar los estudios acerca de un conflicto mayor como lo representa la independencia cubana.
\end{abstract}

\section{Palabras clave}

Insurrección; Cuba; España; Arístides Agüero; Partido Revolucionario Cubano

\begin{abstract}
This article aims to analyze the networks developed by the Cuban Revolutionary Party on Peru, Chile and Bolivia, with the intention of obtaining support for the Cuban insurrection of 1895 . Within the studies on the war of 1895 there is evidence of an absence in regarding to diplomatic relations on the part of the Republic of Cuba. Through public and private correspondence it will be demonstrated, then, how one of the delegates of that party, Arístides Agüero, builds power relations through various actions on the Southern Andean Region. Considering war as a complex social fact, the aim is to describe the impact of networks by locating their areas of realization, whether they are organizational, political or military. Likewise, it seeks to complement studies on a greater conflict as represented by Cuban independence.
\end{abstract}

\section{Keywords}

Insurrection; Cuba; Spain; Aristides Agüero; Cuban Revolutionary Party

Esta obra está sujeta a la Licencia Reconocimiento-NoComercial-CompartirIgual 4.0 Internacional de Creative Commons. http://creativecommons.org/licenses/by-nc-sa/4.0/ 


\section{Claudio Antonio Gallegos}

\section{Introducción}

El siglo XIX refiere, entre otros aspectos, a las luchas revolucionarias del continente americano en búsqueda de culminar con los lazos coloniales con la monarquía española. Parecería que 1824 plantea el fin de este proceso, pero en realidad, según las fuentes trabajadas para este artículo, sólo representa un paréntesis a ser reabierto en las contiendas por la liberación de Cuba y Puerto Rico .

La extensa y variada producción historiográfica del tema analiza este hecho como algo desagregado del resto de las independencias por el solo hecho de ocurrir a destiempo. Para 1868 y, aún más para 1895 y 1898, Nuestramérica está compuesta por una amplia gama de jóvenes Estados en estrecha relación con las potencias europeas.

En este mismo sentido, la mayoría de los estudios han ignorado la preocupación de las fuerzas insurrectas cubanas en búsqueda del apoyo y legitimación del acto libertario por parte del resto de las naciones del continente, como fragmento del escenario regional de desarrollo del conflicto.

En contrapartida, es posible encontrar una amplia gama de escritos referentes a la extensa red de apoyo a la causa española frente a los conflictos suscitados en Cuba. Pero la presencia de enviados plenipotenciarios del Partido Revolucionario Cubano (en adelante PRC) por Nuestramérica referencia escasos estudios.

El mentado partido fue fundado en enero de 1892 con el objetivo, entre otros, de instaurar en el exterior una extensa red de apoyo y legitimación a la causa independentista por medio de la creación de clubes, agencias, asociaciones, etc. Los primeros fueron creados en Estados Unidos pero con posterioridad también se desarrollaron por el resto del continente.

Estas acciones dan cuenta de la necesidad de expandir la guerra más allá del campo de batalla: se espera una guerra desde dentro y fuera de la isla. A diferencia de la primera guerra por la Independencia de Cuba, en la Revolución de 1895 habría un plan ideado para llevar a cabo nuevas relaciones con el resto de los estados.

En este contexto es que entre 1895 y 1898, Arístides Agüero lleva a cabo un viaje diplomático por el sur del continente para tratar el proceder de las naciones del sector en cuanto al proceso independentista cubano. De los países que recorre, haremos especial énfasis en los enmarcados dentro de la Región Andina Sur (Chile, Bolivia y Perú), debido a que este artículo representa una parte de una investigación mayor enmarcada en organismos de Ciencia y Tecnología de la República Argentina. El objetivo del presente trabajo, entonces, radica en rastrear la conformación de las diversas redes gracias al viaje de Agüero, los actores que formaron parte de ellas y sus acciones, así como también describir el impacto de las mismas, localizando sus ámbitos de realización, ya sean organizacionales, políticos o bélicos.

La documentación para el estudio que se plantea se basa en epistolario, diarios de campañas, censos poblacionales, diarios de guerra, anales de información y medios gráficos de prensa, entre otros. En este sentido queda claro que la heurística abre el camino de análisis. Las fuentes son indagadas desde el análisis crítico del discurso, 


\section{La Revolución cubana de 1895. El aporte de los viajes de Arístides Agüero a Perú, Chile y Bolivia.}

considerando su contenido. Las mismas fueron obtenidas y trabajadas, en forma personal, en archivos ubicados en Argentina (Biblioteca Nacional Argentina), España (Archivo del Ministerio de Asuntos Exteriores y de Cooperación de España; Archivo Histórico Nacional; Archivo de la Administración de Alcalá de Henares), Cuba (Biblioteca Nacional de Cuba "José Martí") y Estados Unidos (Columbia University Libraries; New York Public Library y National Archives at Washington, DC), algunas de los cuales son citadas en el cuerpo de este artículo.

Sobre las mismas recaen diversas preguntas de investigación entre las que se destacan: Durante la revolución de 1895 ¿es posible dar cuanta de una programación de actividades por parte del PRC en busca de apoyo a la causa independentista cubana por parte de los Estados de Nuestramérica? Y si éste fuera el caso ¿Quiénes realizaron dichas tareas? ¿De qué manera fueron llevadas a la práctica? ¿Es posible evidenciar éxitos o fracasos en el análisis del accionar de los enviados de Cuba?

Estas son solo algunas de las cuestiones que es necesario analizar para poder comprender un aspecto de esta guerra escasamente trabajado, sobre el que es necesario profundizar a fin de complementar los estudios existentes.

Para ello se ha estructurado este artículo en dos partes: primero, una breve contextualización histórica sobre el proceso que se pretende abordar que representa el marco de acción del PRC para el envío de delegados, haciendo especial hincapié en Arístides Agüero y, en una segunda parte se da cuenta del periplo del mencionado actor sobre Perú, Chile y Bolivia.

\section{Contextualización}

Guillermo Moncada sobre la zona de Santiago, y Bartolomé Masó por el río Cauto, inician el 24 de febrero de 1895 un nuevo levantamiento en la isla de Cuba, específicamente sobre el Oriente, que se conocerá como "Grito de Baire".

Este alzamiento intentó ser exitoso en diversas poblaciones de la isla pero no tuvo en todos lados la misma suerte. Claro ejemplo de ello lo referencia el fracaso de las acciones sobre Occidente como consecuencia del apresamiento de dos de los principales líderes del momento por parte de las fuerzas españolas: Julio Sanguily y José María Aguirre .

Casi como un reflejo de lo ocurrido hacia 1868, la insurrección daba cuenta de algunos rasgos de inmadurez a ser subsanados. De este modo, el foco de los enfrentamientos se direccionó sobre el Oriente de la isla.

Un mes después del “Grito de Baire”, José Martí, como delegado del PRC y Máximo Gómez, como General en Jefe del Ejército Libertador, respectivamente, firman el "Manifiesto de Montecristi", en alusión a la localidad dominicana en donde fue sellado el mismo. Resulta pertinente citar el primer párrafo del mismo:

\footnotetext{
"La revolución de independencia, iniciada en Yara después de [s] preparación
} gloriosa y cruenta, ha entrado en Cuba en un nuevo período de guerra, en virtud 


\section{Claudio Antonio Gallegos}

del orden y acuerdos del Partido Revolucionario en el extranjero y en la Isla, y de la ejemplar congregación en él de todos los elementos consagrados al saneamiento y emancipación del país, para bien de América y del mundo; y los representantes electos de la revolución que hoy se confirma, [sus títulos] reconocen y acatan su deber, -sin usurpar el acento y las declaraciones sólo propias de la majestad de la república constituida,-de repetir ante la patria, que no se [debe] ha de ensangrentar sin razón, ni sin justa esperanza de triunfo los propósitos precisos, hijos del juicio y ajenos a la venganza, con que se ha compuesto, y llegará a su victoria racional, la guerra inextinguible que hoy lleva a los combates, en conmovedora y prudente democracia, los elementos todos de la sociedad de Cuba" (Martí, 1975, p.93).

Explícitamente deja en claro la necesidad de establecer la idea de la guerra incluso en el extranjero, considerando, también, que la misma no debe estar teñida de odio, cólera o venganza.

El ideólogo de la gesta de 1895 contaba con el apoyo de los sectores más radicales de la sociedad cubana, compuesto por trabajadores comprometidos en llevar a cabo un proceso democrático revolucionario y de liberación nacional. Son premisas de este grupo el respeto por el republicanismo y la democracia, vinculadas a la idea de conformar la unidad de los pueblos de Nuestramérica junto a un explícito rechazo a injerencias de tipo imperialistas en la zona. Sin embargo, José Martí muere en combate el 19 de mayo de 1895, y a partir de ese momento la revolución debe rediseñar su accionar producto de la muerte de su líder.

El 16 de septiembre de 1895, en la población de Jimaguayú, en Camaguey, se congregaron los representantes de los distintos grupos de rebeldes para dar una nueva Constitución republicana a la Cuba Libre y designar un gobierno. La breve Constitución de Jimaguayú, descartando la formación de una asamblea legislativa permanente, creó un simple Consejo de Gobierno, compuesto por un Presidente y un Vicepresidente -lo serían Salvador Cisneros Betancourt y Bartolomé Masó.

En lo que respecta a los sucesos en la isla, luego de darse a conocer dicha carta orgánica, Antonio Maceo, como uno de los Jefes de las fuerzas insurrectas se instala en el oriente mientras que Máximo Gómez hace lo propio sobre la zona de Las Villas. Luego de este reordenamiento estratégico, cubanos y españoles se enfrentan en Iguará, con diferencia favorable para los cubanos.

Los primeros meses son beneficiosos para los insurrectos, pero el esfuerzo de la guerra deja como resultado tropas exhaustas y mala alimentación, sumado a inconsistente armamento. Es por ello que la nueva estrategia no es defensiva sino ofensiva.

El fuerte español ubicado en cercanías de Mal Tiempo es atacado por las fuerzas cubanas logrando el éxito esperado en lo que respecta a obtención de alimentos, armamentos y municiones. De esta manera se revitaliza el ejército con miras a continuar su plan de despliegue hacia el oeste. Si bien permanecen con buena racha al poder atravesar la columna de Matanzas, luego optan por la división de filas para ingresar en Caliseo, y es en ese momento donde deben retirarse al enfrentarse a un superior ejército español, muy bien organizado y preparado para dar batalla. Es 


\section{La Revolución cubana de 1895. El aporte de los viajes de Arístides Agüero a Perú, Chile y Bolivia.}

dable recordar que esas tropas realistas se encuentran comandadas por Martínez Campos.

En enero de 1896 se reúnen en La Habana Máximo Gómez y Antonio Maceo con el fin de coordinar la marcha de la guerra. El primero de ellos se dirige hacia la provincia de Pinar del Río, mientras que el segundo permanece en La Habana desarrollando una táctica defensiva.

Con la idea de atravesar la fuerte Trocha de Muriel, parte Maceo. Logra llegar a occidente y se sitúa en Matua. Habían transcurrido 90 días en los que, según Foner (1795):

\footnotetext{
“Se habían cubierto 1696 kilómetros, se disputaron 27 batallas, se tomaron 22 ciudades importantes, se capturaron más de 2000 rifles, 8000 cartuchos de munición y 3000 caballos. Una hazaña impensable para un puñado de patriotas cubanos que se enfrentaron a 124 batallones de infantería, 40 escuadrones de caballería, 16 baterías de artillería de campaña, 6701 generales y otros oficiales, 183571 tropas individuales en línea, más de 60000 voluntarios y guerrilleros y un sistema de trochas" (p. 95).
}

Los inesperados éxitos cubanos según las fuerzas españolas, dieron lugar a un nuevo cambio de mando y es por ello que Martínez Campos, quien dimitió de su puesto, fue suplantado por Valeriano Weyler. El nuevo gobernador de Cuba tiene como objetivo poner fin a la insurrección y pacificar la isla. Por ello recibe un refuerzo de 3000 hombres para combatir con las fuerzas ofensivas comandadas por Maceo, las cuales referenciaban un número menor.

Los meses de agosto, septiembre y octubre de 1896 presentan diversidad de batallas con triunfos para ambos bandos. Entre ellos se recuerda la lucha en Montezuelo, Tumbas de Estorino, Ceja del Negro, entre otras.

Debido a conflictos internos en lo concerniente a las decisiones de gobierno en esta segunda etapa de la República de Cuba en Armas, Gómez solicita la presencia de Maceo en La Habana. En el tránsito a dicha ciudad se enfrenta a las fuerzas españolas quienes provocan su muerte.

El 7 de diciembre de 1896 la insurrección sufre un nuevo golpe con la desaparición de Maceo, habiendo transcurrido recién un poco más de un año de la muerte de Martí. Más allá de estos desafortunados hechos para los cubanos, se percibe un incremento en las ansias de libertad observable en la continuación de la guerra.

La política de España se recrudeció con mucha intensidad. Prueba de ello fue la implementación de la reconcentración de población, que dejó efectos devastadores para los cubanos, dando cuenta de una crueldad quizá inusitada para ese momento. Así, la revolución de a poco se fue diluyendo producto también de la puesta en funcionamiento de la autonomía en Cuba para asuntos domésticos en 1897 por medio de Ramón Blanco, nuevo representante español.

Ahora bien, el 19 de septiembre de 1895 en la Asamblea de Jimaguayú a la que se hizo referencia con anterioridad, fue elegido Tomás Estrada Palma como Delegado Plenipotenciario del gobierno provisional de Cuba. Sin embargo, la misión de Agüero 


\section{Claudio Antonio Gallegos}

fue aprobada con anterioridad a esta fecha -que no le daba a Estrada Palma la potestad de elegir agentes de la república- por lo que, su viaje es de carácter partidista y no gubernamental. Incluso recién el 21 de noviembre de 1895 Arístides Agüero recibe, oficialmente, las atribuciones de delegado.

Según José Martí (1892), el lugar primordial para conseguir el apoyo y la legitimación a la causa de Cuba eran los países de Nuestramérica. Sostenía que eran necesarias acciones sobre esas geografías "a fin de acelerar -con menos sangre y sacrificios- el éxito de la guerra y la fundación de la nueva República indispensable al equilibrio americano" (pp. 96-97).

Pero la realidad daba cuenta de que los jóvenes estados en plena etapa de conformación y consolidación intentaban robustecer sus relaciones con las naciones de Europa, entre las que claramente España jugaba un importante rol.

En este sentido, la mayor parte de las cancillerías de las naciones del sur del continente apelaron, hacia 1895, a la neutralidad en lo concerniente al enfrentamiento entre Cuba y España. La añeja potencia europea jugaba un nuevo rol sobre Nuestramérica continental, pasando de lazos de coloniaje a relaciones económicas y políticas de tipo modernas, dando pasó, asimismo, a un marcado hispanismo ferviente.

Claramente lo expresaba Arístides Agüero desde Chile en carta enviada a Tomás Estrada Palma el 11 de abril de 1896:

\footnotetext{
"Estas repúblicas tiene todavía gran respeto a la antigua señora y dueña y esto lo disfrazan de dos modos, ya fingiendo un amor a la madre patria por ser tan desgraciada, la misma raza, etc., ya diciendo, que no pueden crear a su país nuevas complicaciones internacionales, etc., etc." (Correspondencia, 1943, t. II, p. 39).
}

Incluso en uno de sus informes finales, el mencionado delegado cubano comenta su parecer respecto de los países del sur en carta fechada 22 de mayo de 1898:

\footnotetext{
"Respecto a la cuestión cubana en Sud América le voy a dar una ligera reseña del estado de la opinión. Brasil favorable a nosotros; pero no reconocerá -por ahora- pues, limitará los yankees en su última resolución. Uruguay hostil a los yankees no reconocerá por las razones que el año pasado le expuse más las simpatías españolas en la enemistad yankee. Argentina y Chile hostiles a Washington hemos perdido mucho terreno y las simpatías a España aumentan cada día. La guerra entre ambos está sobre el tapete aun. Perú, Bolivia, Ecuador -francamente partidarios de España- tienen un arbitraje de la reina regente y por nada nos reconocerán hoy ni mañana. En resumen no creo nos reconozcan ningún país latinoamericano, unos por simpatías españolas, otros por antipatías yankees y otros por apatía sempiterna" (Correspondencia, 1943, t. II, p. 145).
}

Puede considerarse al viaje de Arístides Agüero, entre 1895 y 1898, como uno de los más destacados trabajos diplomáticos por la independencia cubana. Hombre joven, parte de una familia con extensa participación en la vida sociopolítica de la Isla. La misión que comandaba, como delegado del PRC, buscaba lograr un plan de acción 


\section{La Revolución cubana de 1895. El aporte de los viajes de Arístides Agüero a Perú, Chile y Bolivia.}

entre cuatro repúblicas: Perú, Chile, Bolivia y Ecuador. Para este periplo se designó a Nicolás de Cárdenas como su compañero de viaje. El esquema de su plan de acción se basó en organizar comités cubanos en todas las regiones; celebrar conferencias y mítines en todas las ciudades que se pueda; obtención de peticiones departamentales en favor de la beligerancia dirigidas a las Cámaras y al Ejecutivo y ganar votos en las cámaras para la aprobación de esas peticiones populares (Morales \& Sánchez, 1998).

El objetivo principal, lograr apoyo y legitimación a la causa más la recaudación de dinero, fue acompañado de un documento de suma importancia para la época: “Cuba contra España", escrito por Enrique José Varona. El mismo fue concebido como un manifiesto del Partido Revolucionario Cubano a los pueblos de Hispanoamérica. Entre sus líneas se puede ver la importancia de la legitimidad de la rebelión cubana contra el dominio español. Como fundamentación a sus afirmaciones se apelaba a cuestiones relacionadas con el mal manejo administrativo y político por parte de la metrópoli.

Arístides Agüero refiere en diversas cartas y de manera recurrente a la obra de Varona, sosteniendo que la misma es de vital ayuda ante el desconocimiento generalizado de la situación en Cuba: "aquí hay desconocimiento completo de nuestra Patria, multitud de preocupaciones y grandes errores sobre nuestra conducta y objetivo. Nada más breve y claro que su obrita, nada más serio y mesurado que sus conceptos" (Archivo Nacional, caja 116, Núm. 311)

Es claro que el trabajo de las misiones diplomáticas del PRC estuvo plagado de inconvenientes y desorden producto de la descoordinación entre este servicio exterior cubano y el desarrollo propio de la guerra en la Isla. Causa de ello fue, entre otros factores, el general desconocimiento de las particularidades de cada uno de los países que se visitó sumado a la escasa experiencia en materia de relaciones internacionales.

La articulación de las acciones en América del Sur se caracterizó por ser lenta. Las particularidades de cada uno de los Estados, lo vasto del territorio y la gran inmigración española en la zona hicieron que el ritmo de trabajo no sea el esperado. Ejemplo de ello fue que Chile esperó a la constitución de la República de Cuba para reconocer a Agüero, a quien consideraban solo un agente de un partido.

\section{Los viajes de Agüero}

Luego de una escala en Panamá, donde logra recolectar algo de dinero, y más allá de las noticias desalentadoras que llegaban a la Isla, Agüero arriba a Perú y es muy bien recibido. Prueba de ello lo representa el lugar que le otorgaron a sus palabras en diversos periódicos locales. Resalta, entre tantos, El Comercio, solidario con la causa cubana desde la guerra de 1868.

No es un dato menor su escala en Panamá. Agüero partió de Cuba el 10 de agosto de 1895 y llega a Colón el 18 de agosto. Allí se encontró con el Dr. Manuel Coroalles, un cubano residente en el Istmo desde hacía varios años, que había logrado desarrollar 


\section{Claudio Antonio Gallegos}

un importante centro farmacéutico. Su paso por este Estado reflejaba la necesidad de recaudar fondos para continuar su viaje por el sur.

Ya en Perú, se encuentra con José Payán, personaje destacado de la Guerra de los Diez Años devenido en exitoso banquero con contactos en el gobierno. Más allá de su postura anexionista, representaba un hombre importante en lo concerniente al desarrollo de estrategias de guerra fuera de la Isla. Su idea era comandar a Agüero en el sur del continente para lograr un acuerdo diplomático secreto de apoyo a la causa cubana.

Pero Perú para 1895 vivía momentos de tensión. Otra revolución, distinta a la encarada en Cuba, enfrentaba a Cáceres y De Piérola. Gran cantidad de pérdidas humanas en distintas batallas sangrientas dejaron como saldo la victoria de De Piérola, dando inicio a una nueva etapa en la historia peruana.

De Piérola en el poder representaba la unión entre la oligarquía guanera, la jerarquía eclesiástica y la clase media. Estos últimos resaltan sus tradiciones y orgullo hispánico y católico.

Antes de que Agüero se entreviste con el Presidente realiza, junto a su compañero Cárdenas, una serie de actividades de propaganda y organización. En septiembre, el Comité Patriota Cubano, formado por un reducido número de emigrantes, recibe a los agentes del PRC y en conjunto deciden re-nombrar a la agrupación como Leoncio Prado.

Las actividades continuaron buscando presencia en la prensa peruana. Recorrieron los siguientes periódicos: El Comercio, La Opinión Nacional y El Tiempo. Logran concertar canjes con los periódicos cubanos Patria y El Porvenir. Esto daría inicio a relaciones formales con corresponsales y citación de fuentes.

También se encuentran con jóvenes estudiantes y obreros concentrados en el Club de los Estudiantes y la Unión Federal de Obreros. Con ellos se organiza un mitin procubano. Es preciso recordar que dentro del plan de acción se sugería la posibilidad de organizar clubes de mujeres, sobre todo en Lima, con el objeto de buscar fondos destinados, pura y exclusivamente, a reunir auxilios para los heridos de guerra.

El 12 de septiembre se reconstruye el Club Lima, presidido por Manuel Portuondo. Cuenta con la presencia de Juan Dunay como tesorero y Francisco López Chávez como secretario. También se crea un club compuesto sólo por peruanos y se lo denomina Club Independencia de Cuba, con fuerte presencia de los estudiantes.

La realidad era que la emigración cubana por Perú era escaza, las recaudaciones no eran abundantes, pero sí se había logrado una presencia considerable en la opinión pública. Esto último dio lugar a una reunión con el Presidente.

Luego de la visita a De Piérola se decidió que Nicolás de Cárdenas permaneciera en Lima mientras que Arístides Agüero continuara su periplo hacia Chile. Antes de su partida, la Delegación en Nueva York recibe un resumen de las actividades realizadas por medio de una carta que envía el 16 de septiembre de 1895:

“ $1^{\circ}$ Hemos organizado el Club cubano Leoncio Prado: de ello recibirá nota por la directiva. 


\section{La Revolución cubana de 1895. El aporte de los viajes de Arístides Agüero a Perú, Chile y Bolivia.}

$2^{\circ}$ provocamos erogación cubana, el club dio 500 soles, Payán 750 y su cuñado 100; total 1350 soles. La erogación peruana aún está en trabajo, será de obreros, estudiantes y particulares.

$3^{\circ}$ Sesión en Unión Federal de Obreros; aquí obtuvimos, previos discursos, y, que den algún dinero, pasen telegramas a las demás sociedades obreras del Perú invitándolas con el mismo fin y por último un acuerdo con los estudiantes para hacer una velada musical a beneficio de Cuba.

$4^{\circ}$ Sesión en el Consistorio Carolino de los estudiantes limeños, prometieron acudir a las demás universidades, trabajar veladas de común acuerdo con los obreros y hacer erogación por Cuba.

$5^{\circ}$ Constitución de un club de peruanos Independencia de Cuba en el Callao (sic); trabajará propagando y recolectando fondos

$6^{\circ}$ Hemos comenzado a tratar de formar un depósito de armas, aquí ofrecen muchas los particulares y es probable que por alto conducto se nos de mayor número de ellas; al presente podemos contar 40 Manlicher y sin otro auxilio superior espero llegarán a 100.

$7^{\circ}$ Hemos visitado los personajes más elevados de este país y algo se puede esperar. Payán les dará más detalles luego.

$8^{\circ}$ Visitamos y facilitamos a la prensa todos los periódicos que de allí recibimos; está muy animada por nuestra causa" (Correspondencia, 1943, t. II, pp. 22-23)

La cita anterior evidencia la conexión entre los delegados y la sede del PRC en Nueva York, como parte de una estrategia. Luego de la recepción de dicha carta, el periódico cubano Patria reprodujo la editorial de su par peruano El Comercio, donde se destacaron las exitosas intervenciones tanto de Agüero como de Cárdenas.

Asimismo, comenzaron a aparecer entre las columnas del periódico peruano algunos extractos de Patria, como ocurriera también con su par de la colonia italiana en Perú La Voce d' Italia.

La idea rectora de formar un acuerdo diplomático con Perú, Chile y Bolivia conoce su primer traspié. El paso por Lima no tuvo como resultado la firma de las autoridades del gobierno. De todas formas, Perú representaba el país con mayor apoyo a la causa cubana, seguido por Chile y Brasil.

La negativa por parte de Perú a realizar acuerdos formales tiene como origen los problemas limítrofes de esta nación con Ecuador. Será la corona española la encargada de actuar como árbitro en esta contienda. Es por eso que se permite el ingreso de la misión del PRC y la realización de actividades pro-cubanas siempre y cuando esto no genere conflicto en la sociedad. En la correspondencia diplomática entre cubanos puede leerse en reiteradas oportunidades este pedido por parte del gobierno peruano.

Con un poco más de 1000 soles, recolectados en distintas actividades, Agüero continúa su misión con destino a Chile.

El primer contacto con el país transandino lo tiene en la ciudad de Iquique, como paso para poder llegar a Tarapacá, lugar de desarrollo de uno de los primeros clubes patrióticos, fundado por Antonio Rosado.

Al igual que en Perú, la emigración patriótica por la zona no representaba un número realmente considerable. Según el intercambio epistolar, la colonia cubana en Santiago de Chile no llegaba a quince miembros. Sin embargo, había sido posible la 


\section{Claudio Antonio Gallegos}

conformación de una serie de clubes pro-cubanos tales como el Comité Internacional Republicano Independiente de Cuba; la Sociedad Sudamericana de Señoras $\mathrm{N}^{\circ} 1$ y el Club Revolucionario Cubano. Unido a ello, medios gráficos entre los que se destacan El Americano (bisemanario) y La Ley referenciaban la causa cubana de manera positiva (Gallegos, 2017, p.32).

De todas formas, las diversas acciones realizadas para conseguir apoyo económico dieron como saldo la obtención de 6000 pesos chilenos, los cuales tenían como destino la ciudad de Nueva York. Desde allí se organizaba el financiamiento de las acciones realizadas en la isla.

La cantidad de dinero mencionada no era menor, así lo da a entender Arístides Agüero en sus correspondencias, destacando la capacidad organizativa para lograr un gran éxito en un muy corto período de tiempo.

A partir de ese momento, Chile comienza a ser considerado un excelente lugar para desarrollar estrategias tendientes a lograr los objetivos propuestos en la misión. Da cuenta de la potencialidad que demuestra el país y llega a sostener "aquí hay dinero como en ningún otro lugar" (Morales \& Sánchez, 1998, p. 260).

Los actores chilenos de esas acciones provenían de los sectores obrero y artesano de la ciudad. Asimismo ellos llevaban adelante las tareas de propaganda por la causa de Cuba en los medios que tenían a su alcance. Solo por nombrar un caso, la Confederación Obrera en coordinación con una variedad de asociaciones y en compañía de estudiantes sobre todo universitarios, dieron lugar a la realización de diversos actos en favor de la independencia de Cuba.

Al igual que en Perú, la colonia italiana abraza los ideales de liberación. La Sociedad Republicana Socialista Giuseppe Mazzini organiza un comité pro-cubano y recauda fondos. Se constituye también el Comité Internacional Republicano Independiente de Cuba. Dicha entidad organiza una actividad solidaria con Cuba en el Teatro Municipal del Puerto y a la misma concurren 400 personas.

Con este panorama se encontró Agüero a su llegada. Es por ello que la misión diplomática comienza a centrarse más en este país que en Perú, como se había pensado en un primer momento. Es menester destacar también el trabajo de Eugenio María de Hostos y Nicolás Tanco, como fervientes defensores y propagadores de las gestas libertarias.

Agüero también desarrolla relaciones con la prensa a fin de poder operar sobre la opinión pública. En este sentido toma contacto con periódicos tales como El Americano, La Ley, La Nueva República, Democracia, Revista Cuco, Revista Cómica y El Heraldo.

La prensa chilena, hasta el momento del arribo de Agüero, se caracterizaba por dar a conocer partes de guerra, por ejemplo, sin exponer algún tipo de preferencia en el conflicto. Pero la presencia de este delegado en el país puede ser un punto a considerar al ver que esos medios de prensa comienzan una campaña de búsqueda de armamento.

Por otro lado, Agüero tuvo que hacer frente a aquellos que no comulgaban con su causa. En carta enviada a Tomás Estrada Palma el 16 de octubre de 1895 sostiene 


\section{La Revolución cubana de 1895. El aporte de los viajes de Arístides Agüero a Perú, Chile y Bolivia.}

que "los demócratas, radicales, obreros y estudiantes están con nosotros, pero los conservadores y clericales nos hacen la guerra sorda" (Correspondencia, 1943, t. II, p. 27).

La denominación "guerra sorda" era utilizada para caracterizar los bandos desde posturas incluso culturales. En este sentido, España representaría el catolicismo, la gallardía, la Madre Patria, la cultura y la raza. En contrapartida, Chile quedaba en el lugar de nación racista, criminal y anti-papal al apoyar y defender una causa en contra de España. También se debe destacar que los defensores de los derechos españoles sobre Cuba que se encontraban en Chile denostaban la presencia de Estados Unidos, a quienes la adjetivan como intrusa.

En la última parte de su periplo, Agüero visitó Viña del Mar, Valparaíso, Limache y Quillota, para pasar finalmente por Talca, Concepción y Aranco. Este trayecto deja como saldo una recaudación inesperada de 18000 pesos chilenos.

Son claros los primeros logros: apoyo por parte de varios medios gráficos de prensa, desarrollo de un mayor número de actividades pro-cubanas fomentadas desde la Confederación Obrera e incluso la presencia de varios militares chilenos dispuesto a prestarse como voluntarios en la guerra en Cuba.

Es necesario destacar en este momento que Arístides Agüero, junto al puertorriqueño Eugenio María de Hostos, fue el principal reclutador de soldados chilenos dispuestos a viajar a Cuba para unirse a las fuerzas insurrectas. Se han logrado reconocer alrededor de 20 personas provenientes de Chile en el Ejercito libertador cubano (García del Pino, 1996).

De esta manera, es posible referenciar una variedad considerable de chilenos que se alistarán para luchar por Cuba entre los cuales se destaca Pedro Vargas Sotomayor. A la temprana edad de 15 años, Sotomayor asiste a un mitín acerca del fin del coloniaje español en Cuba. Para 1895 viaja a la isla, específicamente a la ciudad de Santiago donde toma contacto con Antonio Maceo por recomendación de Agüero. Su desempeño sorprende al líder cubano a punto tal de nombrarlo maestro de mambises.

Fue partícipe de la recordada batalla de Mal Tiempo el 15 de diciembre de 1895 donde es derrocada una columna española produciendo considerables bajas en el ejército enemigo. Un año después, integró el ejército que intervino en la batalla de Ceja del Negro frente a las tropas de Valeriano Weyler. En este contexto, y de manera repentina, fallece Sotomayor a causa de una enfermedad psiquiátrica. Los datos acerca de este deceso son variados y contradictorios por lo cual resulta complejo establecer, incluso, el lugar de su muerte.

Recapitulando, el objetivo de lograr el reconocimiento formal y público de la beligerancia en la isla no fue cumplido en Chile, como así tampoco en la mayoría de las jóvenes naciones del sur del continente.

Si bien desde la isla se pensaba que Chile o Venezuela podrían dar el primer paso esto no ocurrió. Y en este caso nuevamente los hechos domésticos se entrelazan con los cubanos. Chile se encontraba con la amenaza de un conflicto armado con la República Argentina en cuanto a los desentendimientos públicos en temas 


\section{Claudio Antonio Gallegos}

fronterizos, lo que llevó al gobierno del primero de ellos, e incluso también al segundo, a una postura neutral frente al conflicto que enfrentaba a Cuba con España. La posibilidad que en este caso también fuera la corona española la encargada de dirimir en el asunto de las naciones del sur pone freno a los gobiernos en cuanto a apoyos explícitos.

La misión en Bolivia se modificó en su propio desarrollo. Las localidades seleccionadas para visitar fueron La Paz y Cochabamba. Pero en un informe a Estrada Palma del 4 de enero de 1896, el delegado Agüero comenta que una persona cercana al presidente, que no nombra, le advierte: "Sr. Agüero, creo será conveniente marche Ud. por donde vino, si Ud. visita La Paz y Cochabamba, temo haya motines, etcétera, porque el partido oposicionista está haciendo política con Cuba y creo prepárase una asonada al Gobierno" (Archivo Nacional, caja 309, núm. 28).

Finalmente Agüero desiste de la idea de visitar dichas ciudades, donde casualmente llegarían representantes de España para participar en diversos actos. Ante la posibilidad de ser expulsado, el delegado cubano opta por no viajar.

Hacia el mes de agosto de 1896 Arístides Agüero emprende su viaje a Bolivia. Llega a Sucre el 15 de agosto y comienza con sus tareas de delegado del PRC. En ese entonces había sido recientemente elegido como Presidente el conservador Severo Fernández Alonso, con quien Agüero se reúne. Asimismo, poseía una serie de cartas para el jefe del partido liberal boliviano, José Manuel Pando, pero en este caso no se concretó la reunión.

La particularidad de las acciones en Bolivia radica en la ausencia de emigración cubana. Agüero, en reiteradas oportunidades, resalta en su correspondencia su tarea propagandística solitaria, sin ayuda de compatriotas. Se suma a esto la pobreza característica de la zona que tiene como resultado una colecta insignificante. En contrapartida, la presencia española demuestra riqueza y buenas relaciones con el poder.

Tampoco se encuentran canales de legitimación y apoyo a la causa cubana en la prensa antes de la llegada del enviado del PRC. El periódico La Industria referencia los hechos cubanos muy esporádicamente.

Ya como una constante propia de la zona, las cuestiones limítrofes representaban el centro de la atención de las Relaciones Internacionales del país. Cuestión que se veía reflejada, también, en el expansionismo norteamericano. La línea pan hispanista, que se enfrenta a la panamericana, logra varios adeptos a la causa, revigorizando aún más la defensa a España.

Más allá de los inconvenientes, Arístides Agüero logra formar el Comité Central Cubano con presencia de destacados políticos del momento pertenecientes a distintas fuerzas. Es menester destacar que el presidente honorario del mismo era Gregorio Pacheco, dueño de una gran cantidad de minas de plata. Acto seguido se llevó a cabo un mitín en donde se dio lectura a una solicitud popular dirigida al gobierno buscando su apoyo a la lucha en Cuba. Diez días después, se realizó otra reunión, con mayor concurrencia, en donde se expuso un resumen de las acciones cubanas frente a España desde 1821. Se aclaraba que los españoles no habían podido 


\section{La Revolución cubana de 1895. El aporte de los viajes de Arístides Agüero a Perú, Chile y Bolivia.}

triunfar sobre la insurrección y que todas las provincias estaban sublevadas. Asimismo se reconoce la beligerancia de los cubanos y el apoyo de Bolivia a la causa independentista.

Sucre, inesperadamente, se convierte en una ciudad de considerable apoyo. Antes de la partida de Agüero a Potosí, se envía a las Cámaras y al Ejecutivo un acta de los mítines realizados. En ella se dejaba en claro, también, la presencia de un gobierno cubano en la isla, con su propio ejército, recaudación de impuestos, y varios representantes en el exterior.

Ya en Potosí, la misión buscaba, por un lado, recolectar fondos en una zona caracterizada por el desarrollo acuífero y, por el otro lado, dar a conocer la petición de Sucre. Para reforzar las acciones se crearon comités departamentales coordinados desde Sucre.

El saldo termina siendo positivo en lo que respecta a peticiones departamentales que reconocen la beligerancia de los cubanos. Las recaudaciones no tuvieron la misma suerte.

Es necesario dar a conocer también la escasa presencia de fuentes que refieran a esta temática en particular. Tanto periódicos locales como otro tipo de fuentes públicas y privadas brindan escasos datos para reconstruir las redes el PRC en Bolivia. Más allá de eso, la información existente da cuenta de un panorama general en el país que permite realizar las aseveraciones que fueron vertidas en este artículo.

\section{Conclusiones}

El plan del PRC por América del Sur, en la práctica, modifico sus actividades en función de las particularidades propias de los lugares que se visitó. En un primer momento, ante los diversos escollos que fueron expresados en el cuerpo de este trabajo, se consideró como difícil lograr los objetivos de la misión. Así lo comunica Agüero:

"Creo como usted que necesitamos predicar mucho -aún a los sordos-, pero tengo la convicción de que la América nos abandona. El pueblo mucho entusiasmo, las clases altas retraídas: enemigas, los gobiernos indiferentes, tímidos y sin altura de miras" (Archivo Nacional, caja 116, núm. 311)

En líneas generales es posible divisar éxitos y fracasos en distintas zonas, sin que una se caracterice por sobre la otra por algo en especial. Tanto comités, asociaciones o clubes y la prensa local desarrollan diversas actividades en donde el éxito es aleatorio.

Una única constante lo representa el apoyo de los representantes de la comunidad italiana en los distintos países estudiados. Los referenciados en este trabajo, más otros analizados, dan cuenta del persistente apoyo a la independencia de Cuba por periódicos italianos y distintas Sociedades de Socorros Mutuos. Si bien es clara la necesidad de ahondar en este punto en cuestión, resulta indispensable tomar en consideración el contexto europeo de aquel momento para arriesgar una hipótesis 


\section{Claudio Antonio Gallegos}

que dé cuenta del reiterativo apoyo de la comunidad italiana en Nuestramérica a la insurrección cubana sin dejar de lado la posible necesidad de plantear, sobre todo, un enfrentamiento a España.

Por otro lado, la emigración patriótica representó un número casi insignificante para ser tomado como base de la construcción de redes de apoyo y legitimación antes de la llegada del delegado, salvo en el caso de Perú. En algunos países si había clubes o comités con anterioridad, mientras que en Bolivia las actividades de propaganda fueron desde cero.

La diplomacia de las jóvenes naciones del sur del continente queda expuesta ante la llegada de diversos enviados en general y ante Agüero en particular. Se da cuenta de falta de información e inoperancia producto de la falta de formación. Son varias las cartas, de distintos actores, en donde es posible leer la improvisación a la que se apelaba en materia de política internacional.

También era una realidad que el contexto de los países de Sudamérica no colaboraba con la idea de construir proyectos de unidad. En este sentido se diluye rápidamente la idea de conformar un bloque en el sur, unido y poderoso, que impulsara la legitimidad de las luchas en Cuba, junto al apoyo incondicional de su causa libertaria. Finalmente, el viaje de Agüero colaboró con un importante desarrollo en la opinión pública, la recolección de dinero y armas para la guerra, y el afán de varios militares de enfrentar a España en tierras cubanas.

Retomando lo expresado en la introducción de este trabajo, la idea rectora del PRC giraba en torno de unir el proceso independentista continental al surgido, con posterioridad en el tiempo, en la zona insular. Es por ello que el trasfondo de los apoyos, o la falta de los mismos, debe ser analizado a la luz, por un lado, de las identidades nacionales de los estados a los cuales se visitó y, por otro lado, en relación a la significación de la idea de nación que desde Cuba planteaba la superación de las fronteras estatales y propiciaba una identidad Nuestramericana. Al mismo tiempo, era sencillo suponer la resistencia a la lucha de una causa "ajena" en donde podía perderse incluso la vida.

La presencia de chilenos en las fuerzas revolucionarias cubanas da cuenta de un significado de americanismo vinculado a experiencias más generales en donde la patria convoca, une y propone la lucha para lograr el fin de los lazos coloniales, incluso entregando la vida.

Pero también es dable destacar la estrategia del PRC en esta guerra. Ante la considerable asimetría entre fuerzas, desde el punto que se quiera comparar, obraron en consecuencia logrando que la diplomacia española desarrolle también operaciones más allá de la geografía de contienda.

Esto demuestra que la insurgencia de 1895 sí contaba con planes específicos. Algunos de ellos fueron exitosos y otros no tanto. Vieron que la guerra, en ese momento, iba más allá del enfrentamiento armado e instó a la Madre Patria a enfrentarse en otros territorios y con otras armas. Como se dijo anteriormente, la idea de asimilar la lucha en Cuba a las gestas independentistas de Nuestramérica fue el eje rector. 


\section{La Revolución cubana de 1895. El aporte de los viajes de Arístides Agüero a Perú, Chile y Bolivia.}

Agüero culmina sus viajes entre éxitos y fracasos. El PRC demuestra habilidad en su planteo diplomático. Pero la guerra finalmente contará con otro actor que eliminará la presencia española de sus últimas colonias e implantará una República tutelada en Cuba: Estados Unidos. Otros actores de una misma guerra que va modificando sus formas en tanto el tiempo transcurre.

\section{Bibliografía}

Archivo Nacional de la República de Cuba, Fondos Donativos. Cajas varias.

Blakemore, Harold (1992). Chile, desde la guerra del Pacífico hasta la depresión mundial, 1880-1930. En Bethell, Leslie; Historia de América Latina. Barcelona: Editorial Crítica, Tomo 10.

Casasús, Juan José Expósito. (1953). La emigración cubana y la independencia de la patria. La Habana: Editorial Lex.

Correspondencia de la Delegación Cubana en Nueva York durante la guerra de Independencia de 1895 a 1898. (1943), La Habana: Publicaciones del Archivo Nacional de Cuba.

De Vega, Mariano Esteban, De Luis Martín, Francisco y Morales Moya, Antonio (2004). Jirones de Hispanidad. España, Cuba, Puerto Rico y Filipinas en la perspectiva de dos cambios de siglo. Salamanca: Ediciones Universidad de Salamanca.

Estrade, Paul (1998). El papel de la emigración patriótica en las guerras de independencia de Cuba (1868-1898). Tebeto: Anuario del Archivo Histórico Insular de Fuerteventura. $\mathrm{N}^{\circ} 11$.

Foner, Phillip. (1975). La guerra hispano - cubano - americana y el nacimiento del imperialismo.1895 - 1902. Madrid: Akal editor. Vols. 1 y 2.

Gallego, Tesifonte (1897). La Insurrección Cubana. Crónicas de la Campaña. La preparación para la guerra. Madrid: Imprenta Central de los Ferrocarriles.

Gallegos, Claudio (2017). Internacionalismo revolucionario en América Latina: el accionar de Chile frente al proceso independentista cubano (1868- 1898).

Revista de la Red de Intercátedras de Historia de América Latina Contemporánea. Año 


\section{Claudio Antonio Gallegos}

https://revistas.unc.edu.ar/index.php/RIHALC/article/view/18921/18819

García del Pino, César (1996). Expediciones de la Guerra de Independencia. 18951898. La Habana: Editorial de Ciencias Sociales.

Guerra Vilaboy, Sergio (2000). Los gobiernos hispanoamericanos y la guerra de 1898. Baluarte. Estudios gaditano - cubanos. Número 2.

Hidalgo Paz, Ibrahim (2004). Cuba 1895 - 1898. Contradicciones y disoluciones. La Habana: Centro de Estudios Martianos.

Klarén, Peter (1992). Los orígenes del Perú moderno, 1880-1930. En Bethell, Leslie; Historia de América Latina. Barcelona: Editorial Crítica, Tomo 10.

Klein, Herbert (1992). Bolivia, desde la guerra del Pacífico hasta la guerra del Chaco, 1880-1932. En Bethell, Leslie; Historia de América Latina. Barcelona: Editorial Crítica, Tomo 10.

Lawrence Tone, John (2006). Guerra y genocidio en Cuba: 1895-1898. Madrid: Turner.

Martí, José (1975). Obras Completas. La Habana: Editorial de Ciencias Sociales, tomo 4.

Martí, José (1892). A los presidentes de los Clubs del Partido Revolucionario Cubano en el Cuerpo de Consejo de Key West". Epistolario. Edición Crítica, tomo III.

Morales, Salvador y Sánchez, Agustín (1998). Diplomacias en conflicto. Cuba y España en el horizonte latinoamericano del 98. México: Centro de Investigación Científica Ing. Jorge L. Yamayo A.C.

Naranjo Orovio, Consuelo (2009). Historia de Cuba. Madrid: CSIC - Doce Calles.

Nievas, Flabián (2008). Aportes para una sociología de la guerra. Buenos Aires: Proyecto Editorial.

Pirala, Antonio (1895-1898). Anales de la guerra de Cuba. Madrid: Felipe González.

Torres - Cuevas, Eduardo y Loyola Vega, Oscar (2001). Historia de Cuba. 1492 1898. La Habana: Editorial Pueblo y Educación.

Santovenia, Emeterio (1929). Eloy Alfaro y Cuba, La Habana: Imprenta E Siglo XX. 
La Revolución cubana de 1895. El aporte de los viajes de Arístides Agüero a Perú, Chile y Bolivia.

Scott, Rebecca (1985). Slave Emancipation in Cuba: The Transition to Free Labor, 1860- 1899. Princeton: Princenton University Press.

Recibido: $15 / 07 / 2019$

Evaluado: 20/08/2019

Versión Final: 21/09/2019 